\title{
THE CHEN-TYPE OF THE NONCOMPACT CYCLIDES OF DUPIN
}

\author{
by FILIP DEFEVER, $\uparrow$ RYSZARD DESZCZ and LEOPOLD VERSTRAELEN
}

(Received 6 July, 1992)

Minimal submanifolds of a Euclidean space are contained in a much larger class of submanifolds, namely in the class of submanifolds of finite type. Submanifolds of finite type were introduced about a decade ago by B. Y. Chen in [2]; the first results on this subject have been collected in the books [2], [3].

Let $M^{n}$ be an $n$-dimensional, connected submanifold of the Euclidean space $E^{m}$. Denote by $\Delta$ the Laplacian operator on $M^{n}$, with respect to the Riemannian metric $g$ on $M^{n}$, induced from the Euclidean metric of the ambient space $E^{m} . M^{n}$ is said to be of finite type if each component of the position vector field $X$ of $M^{n}$ in $E^{m}$, can be written as a finite sum of eigenfunctions of the Laplacian operator, that is, if

$$
X=c+\sum_{i=1}^{k} X_{i}
$$

where $c$ is a constant vector, and $X_{1}, \ldots, X_{k}$ are nonconstant maps satisfying $\Delta X_{i}=\lambda_{i} X_{i}$, for $i=1, \ldots, k$. The decomposition (1) is called the spectral decomposition of $X$. If in particular all eigenvalues $\left\{\lambda_{1}, \ldots, \lambda_{k}\right\}$ are mutually different, then $M^{n}$ is said to be of $k$-type. A manifold is said to be of infinite type if it is not of finite type. Let $M^{n}$ be a finite type submanifold whose spectral decomposition is given by (1); if we define a polynomial $P$ by

$$
P(T)=\prod_{i=1}^{k}\left(T-\lambda_{i}\right)
$$

then $P(\Delta)(X-c)=0$.

The class of finite type submanifolds is very large: minimal submanifolds and minimal submanifolds of a hypersphere are of 1-type, compact homogeneous submanifolds, equivariently immersed, are of finite type. Although there are many results on finite type curves and finite type spherical submanifolds, very little is known about the most elementary submanifolds of Euclidean space, namely surfaces in Euclidean 3-space. In particular, no surfaces of finite type in $E^{3}$ are known, other than minimal surfaces, the circular cylinders, and the sphere. In his list [6] of open problems and conjectures concerning submanifolds of finite type, B. Y. Chen mentions the following geometric problem.

Problem. Classify all finite type surfaces in $E^{3}$.

Concerning this problem, we know that minimal surfaces and spheres are finite type (in fact 1-type) surfaces in $E^{3}$. In [4], B. Y. Chen solved the problem for an important class of surfaces, namely tubes. He proved that a tube in $E^{3}$ is of finite type if and only if it is a right circular cylinder $S^{1} \times \mathbb{R}$. Consequently, every closed tube in $E^{3}$ is of infinite type; in particular a circular torus is of infinite type. These cylinders are actually 2-type;

†Supported by a post-doctoral fellowship of the Research Council of the Katholieke Universiteit Leuven.

Glasgow Math. J. 36 (1994) 71-75. 
moreover B. Y. Chen proved [5] that the cylinders $S^{1} \times \mathbb{R}$ are the only null 2-type surfaces in $E^{3}$. Further, in [11], O. J. Garray has shown that a cone in $E^{3}$ is of finite type if and only if it is a plane. More generally, in [8], the problem was solved for the class of ruled surfaces in $E^{3}$. As a generalization of a classical result of Meusnier and Catalan, it was shown that the only ruled surfaces of finite type in $E^{3}$ are the ruled minimal surfaces (i.e. the planes and the right helicoids) and the cylinders $S^{1} \times \mathbb{R}$. Further, in [7], it was shown that the only quadrics of finite type in $E^{3}$ are the circular cylinder $S^{1} \times \mathbb{R}$ and the sphere $S^{2}$; in particular an ellipsoid of finite type in $E^{3}$ is a sphere.

Concerning the noncompact finite type surfaces in $E^{3}$, the preceding results suggest that there are no other noncompact finite type surfaces in $E^{3}$ than the noncompact minimal surfaces and the right circular cylinders. This was already noticed in [8], however the authors hesitated to formulate this as a conjecture.

In 1822, Dupin [9] defined a cyclide to be a surface $M$ in $E^{3}$ which is the envelope of the family of spheres tangent to three fixed spheres. This was shown to be equivalent to requiring that both sheets of the focal set degenerate into curves. The cyclides are equivalently characterized by requiring that the lines of curvatures in both families be arcs of circles or straight lines. Thus, one can obtain three obvious examples: a torus of revolution, a circular cylinder and a circular cone. In turns out that all cyclides can be obtained from these three by inversion in a sphere in $E^{3}$. Finally, the classical cyclides were characterized as the only taut surfaces in $E^{3}$, aside from spheres and planes. For more detailed information on the cyclides, we may refer to [1] and [10]. Here and in the sequel, the term cyclide of Dupin will be used in the narrow sense, i.e. exclusive of the special limiting cases of a sphere, a cylinder, a cone, and a torus.

The noncompact cyclides offer a possibility to break away from a right circular cylinder (2-type) and a circular cone ( $\infty$-type), keeping in common with these surfaces the property that all lines of curvature remain arcs of circles or straight lines. Moreover, the set of noncompact cyclides includes surfaces with singularities (parabolic horn cyclides).

The noncompact cyclides of Dupin are surfaces of the third order and can be parametrized as follows:

$$
\begin{gathered}
x=\frac{V u}{U+V}, \\
y=\frac{U v}{U+V}, \\
z=\frac{V\left(2 u^{2}-a^{2}\right)-U\left(2 v^{2}-a^{2}\right)}{4 a(U+V)},
\end{gathered}
$$

where

$$
\begin{aligned}
& U=\frac{2 u^{2}+a^{2}-d^{2}}{4 a}, \\
& V=\frac{2 v^{2}+a^{2}+d^{2}}{4 a},
\end{aligned}
$$

$a$ and $d$ are parameters. The parameter $d$ corresponds to parallel surfaces. The parameter $a$ corresponds to the semi latus rectum of the focal parabolas. 
The main purpose of this paper is to contribute to the solution of the above problem by showing the following

TheOREM. All noncompact cyclides of Dupin are of infinite Chen-type.

Proof. From the parametrization (3)-(5), we can calculate the components of the induced metric $g_{i j}$, of the inverse metric $g^{i j}$, and $g=\operatorname{det}\left[g_{i j}\right]$ :

$$
\begin{gathered}
{\left[g_{i j}\right]=\left[\begin{array}{cc}
\frac{V^{2}}{(U+V)^{2}} & 0 \\
0 & \frac{U^{2}}{(U+V)^{2}}
\end{array}\right], \quad\left[g^{i j}\right]=\left[\begin{array}{cc}
\frac{(U+V)^{2}}{V^{2}} & 0 \\
0 & \frac{(U+V)^{2}}{U^{2}}
\end{array}\right]} \\
g=\frac{U^{2} V^{2}}{(U+V)^{4}}
\end{gathered}
$$

Hence, the Laplacian $\Delta$ of $M$ can be expressed as follows:

$$
\Delta \equiv \frac{1}{\sqrt{ } g} \frac{\partial}{\partial u}\left(\frac{U}{V} \frac{\partial}{\partial u}\right)+\frac{1}{\sqrt{ } g} \frac{\partial}{\partial v}\left(\frac{V}{U} \frac{\partial}{\partial v}\right)
$$

For later use, we calculate $\Delta x$ and $\Delta^{2} x$, where $x$ is the first component of the position vectorfield of $M$ in $E^{3}$ :

$$
\begin{gathered}
\Delta x=-\frac{u}{U V} \frac{U-V}{U+V} \\
\Delta^{2} x=-\frac{1}{a^{2}} \frac{u}{U^{3} V^{3}(U+V)}\left(a\left(5 U^{3} V-5 U^{2} V^{2}-9 U V^{3}+V^{4}\right)\right. \\
\left.+a^{2}\left(U^{3}-U^{2} V+U V^{2}-V^{3}\right)+d^{2}\left(4 U^{2} V+4 U V^{2}\right)\right)
\end{gathered}
$$

We notice that all terms of $\Delta x$, and $\Delta^{2} x$ are proportional to the expressions of the form $u\left(U^{p} V^{q}\right) /(U+V)$. Therefore we calculate $\Delta\left(u\left(U^{p} V^{q}\right) /(U+V)\right)$.

LEMMA.

$$
\Delta\left(u \frac{U^{p} V^{q}}{U+V}\right)=\frac{1}{a^{2}} \frac{u}{U+V} F(U, V),
$$

with

$$
\begin{aligned}
F(U, V)= & -a\left(p(2 p-1) U^{p+1} V^{q-2}+\left(4 p^{2}+2 p-3\right) U^{p} V^{q-1}+(2 p+1)(p+1) U^{p-1} V^{q}\right. \\
& \left.+q(2 q+1) U^{p} V^{q-1}+\left(4 q^{2}-2 q-3\right) U^{p-1} V^{q}+(q-1)(2 q-1) U^{p-2} V^{q+1}\right) \\
& +\frac{a^{2}}{2}\left(\left((p-1)^{2}+q^{2}\right) U^{p} V^{q-2}+\left(\left(2 p^{2}-2 p-1\right)+\left(2 q^{2}-2 q-1\right)\right) U^{p-1} V^{q-1}\right. \\
& \left.+\left(p^{2}+(q-1)^{2}\right) U^{p-2} V^{q}\right)-\frac{d^{2}}{2}\left(\left((p-1)^{2}-q^{2}\right) U^{p} V^{q-2}\right. \\
& \left.+\left(\left(2 p^{2}-2 p-1\right)-\left(2 q^{2}-2 q-1\right)\right) U^{p-1} V^{q-1}+\left(p^{2}-(q-1)^{2}\right) U^{p-2} V^{q}\right)
\end{aligned}
$$

This follows from (3)-(5) and (10) after a, somewhat long, but straightforward calculation. 
COROLlaRY. For all $k \in \mathbb{N}, \Delta^{k} x$ entirely consists of terms proportional to expressions of the form $u\left(U^{p} V^{q}\right) /(U+V)$.

We notice that $F(U, V)$ is homogeneous of degree $p+q$ in $U, V$, and the parameters $a$, and $d$ (only the parameters which appear explicitly have to be taken into account).

COROLLARY. For all $k \in \mathbb{N}, \Delta^{k} x$ has the following structure

$$
\Delta^{k} x=\frac{1}{a^{2 k-2}} \frac{u}{U+V} G_{k}(U, V),
$$

where $G_{k}(U, V)$ is homogeneous of degree -1 in $U, V$, and the parameters $a$, and $d$.

Since each action of $\Delta$ produces terms of which the degree in the parameters $a$, and $d$ is at most 2 higher, the highest possible degree in the parameters for terms in $G_{k}(U, V)$ is equal to $2(k-1)$, the corresponding degree in $U$ and $V$ of such terms equals $1-2 k$.

Remark. From (11)-(12) and (13)-(14), we notice that $G_{k}(U, V)$ can be written as

$$
G_{k}(U, V)=\frac{P_{k}(U, V)}{U^{2 k-1} V^{2 k-1}},
$$

where $P_{k}(U, V)$ is a polynomial in $U$ and $V$, homogeneous of degree $4 k-3$ in $U, V$, and the parameters $a$, and $d$.

From now on we suppose that $M$ is of $k$-type. Hence there exist numbers $c_{1}, \ldots, c_{k}$ such that

$$
\Delta^{k+1} X+c_{1} \Delta^{k} X+c_{2} \Delta^{k-1} X+\ldots+c_{k} \Delta X=0
$$

This should hold in particular for the first component $x$ of the position vector $X$. Using (15)-(16), we obtain

$\frac{1}{a^{2 k}} \frac{1}{U^{2 k+1} V^{2 k+1}} \frac{u}{U+V}\left[P_{k+1}(U, V)+c_{1} a^{2} U^{2} V^{2} P_{k}(U, V)+\ldots+c_{k} a^{2 k} U^{2 k} V^{2 k} P_{1}(U, V)\right]=0$

From the independence of the functions $\left\{u^{p}\right\}$ and $\left\{v^{q}\right\}$, follows immediately the independence of the set $\left\{U^{p} V^{q}\right\}$. Hence, for (18) to be satisfied, all coefficients of different combinations of powers $U^{p} V^{q}$ in the polynomial

$$
P_{k+1}(U, V)+c_{1} a^{2} U^{2} V^{2} P_{k}(U, V)+\ldots+c_{k} a^{2 k} U^{2 k} V^{2 k} P_{1}(U, V)
$$

should vanish. We focus on those terms of degree $2 k+1$ in $U$ and $V$. Such terms can only occur in $P_{k+1}(U, V)$ since, for $l \in\{0, \ldots, k\}, U^{2 l} V^{2 l} P_{k+1-l}(U, V)$ is homogeneous of degree $4 k+1$ in $U, V$, and the parameters $a$, and $d$, but the highest possible degree in the parameters for terms in $U^{2 l} V^{2 l} P_{k+1-l}(U, V)$ is equal to $2(k-l)$. Amongst the terms of degree $2 k+1$ in $U$ and $V$, we single out the one in $U^{2 k+1} V^{0}$ with the highest possible degree in $U$, and the one in $U^{0} V^{2 k+1}$ with the highest possible degree in $V$. By means of (13)-(14) we can calculate the coefficients of these terms recursively, and as they should vanish, we get the following set of equations

$$
\left\{\begin{array}{l}
a^{2}\left(\frac{a^{2}-d^{2}}{2}+9 \frac{a^{2}+d^{2}}{2}\right) \ldots\left(\frac{a^{2}-d^{2}}{2}+(2 k-1)^{2} \frac{a^{2}+d^{2}}{2}\right)=0 \\
a^{2}\left(9 \frac{a^{2}-d^{2}}{2}+\frac{a^{2}+d^{2}}{2}\right) \ldots\left((2 k-1)^{2} \frac{a^{2}-d^{2}}{2}+\frac{a^{2}+d^{2}}{2}\right)=0
\end{array}\right.
$$


We immediately see that $a=0$ solves this set of equations. We show that $\Sigma_{k}$ does not admit other solutions. The proof goes by induction. The statement clearly holds for $k=1$. We suppose that the statement is true for $k-1$. Suppose also that $\Sigma_{k}$ would have a solution $a_{0}$ with $a_{0} \neq 0$. Then $a_{0}$ cannot be a solution of $\Sigma_{k-1}$. This means that at least one of the expressions

$$
\begin{aligned}
& a_{0}^{2} \ldots\left(\left(a_{0}^{2}-d^{2}\right)+(2 k-3)^{2}\left(a_{0}^{2}+d^{2}\right)\right), \\
& a_{0}^{2} \ldots\left((2 k-3)^{2}\left(a_{0}^{2}-d^{2}\right)+\left(a_{0}^{2}+d^{2}\right)\right)
\end{aligned}
$$

is not zero; so we can divide by this factor in $\Sigma_{k}$. This shows that $a_{0}^{2}-d^{2}$ has to be proportional to $a_{0}^{2}+d^{2}$. Now, there follows easily that $a_{0}^{2}-d^{2}$ and $a_{0}^{2}+d^{2}$ are both zero, and hence $a_{0}=0$; this is a contradiction. We conclude that $\Sigma_{k}$ only admits the solution $a=0$. But $a=0$ corresponds to a complete degeneracy of the focal parabolas, and there does not correspond a cyclide to this situation. This proves our theorem.

\section{REFERENCES}

1. T. E. Cecil and P. J. Ryan, Tight and taut immersions of manifolds (Pitman, 1985). 1984).

2. B. Y. Chen, Total mean curvature and submanifolds of finite type (World Scientific,

3. B. Y. Chen, Finite type submanifolds and generalizations (University of Rome, 1985).

4. B. Y. Chen, Surfaces of finite type in Euclidean 3-space, Bull. Soc. Math. Belg. Sér. B 39 (1987), 243-254.

6. B. Y. Chen, Some open problems and conjectures on submanifolds of finite type, Soochow J. Math. 17 (1991), 169-188.

7. B. Y. Chen and F. Dillen, Quadrics of finite type, J. Geom. 38 (1990), 16-22.

8. B. Y. Chen, F. Dillen, L. Verstraelen and L. Vrancken, Ruled surfaces of finite type, Bull. Austral. Math. Soc. 42 (1990), 447-453.

9. C. Dupin, Applications de géometrie et de méchanique (Paris, 1822).

10. K. Fladt and A. Baur, Analytische Geometrie spezieller Flächen und Raumkurven (Friedr. Vieweg \& Sohn, 1975).

11. O. J. Garray, Finite type cones shaped on spherical submanifolds, Proc. Amer. Math. Soc. 104 (1988), 868-870.

Filip Defever:

INST. v. TheORETISCHE Fysica

Afd. Algebra en Topologie

Celestiunenlaan $200 \mathrm{~B} / \mathrm{D}$

3001 HeVerLeE

BELGIUM

Ryszard Deszcz:

Kastedra MatematyKı

AKademia Rolnicza

ULICA C. NORWIDA 25

50-375 WROCLAW

POLAND
Leopold Verstraelen:

Afd. Algebra en Topologie

Dept. Wiskunde

Celestijnenlaan $200 \mathrm{~B}$

3001 HeVERLEE

BELGIUM 